\title{
Erratum: Extremal limits of the Cvetič-Youm black hole and nilpotent orbits of $\mathrm{G}_{2(2)}$
}

\section{Josef Lindman Hörnlund and Amitabh Virmani}

Physique Théorique et Mathématique,

Université Libre de Bruxelles and International Solvay Institutes,

Campus Plaine C.P. 231, B-1050 Bruxelles, Belgium

E-mail: jlindmane@ulb.ac.be, avirmani@ulb.ac.be

ERRATUM TO: JHEP11(2010)062

In appendix A equation (A.3) the correct expressions for $g_{t \phi_{2}}$ and $g_{\phi_{1} \phi_{2}}$ are as follows:

$$
\begin{aligned}
g_{t \phi_{2}} & =-\frac{2 m \cos ^{2} \bar{\theta}\left[\Sigma\left\{l_{2} c^{3}+l_{1} s^{3}\right\}-2 m l_{1} s^{3}\right]}{\left(\Sigma+2 m s^{2}\right)^{2}} \\
g_{\phi_{1} \phi_{2}} & =\frac{2 m \cos ^{2} \bar{\theta} \sin ^{2} \bar{\theta}\left[l_{1} l_{2}\left\{\Sigma-6 m s^{4}\right\}-2 m\left(l_{1}^{2}+l_{2}^{2}\right) s^{3} c^{3}-4 m l_{1} l_{2} s^{6}\right]}{\left(\Sigma+2 m s^{2}\right)^{2}} .
\end{aligned}
$$

